\title{
LE RÔLE DU VERBE DANS LE RÉSEAU DÉRIVATIONNEL DES PRÉDICATS NOMINAUX
}

\begin{abstract}
Vetulani Grażyna. Le rôle du verbe dans le réseau dérivationnel des prédicats nominaux [The role of verbs in derivation nets of noun-based predicates]. Studia Romanica Posnaniensia, Adam Mickiewicz University Press, Poznań, vol. XXXI: 2004, pp. 459-467. ISBN 83-232-1353-4, ISSN 0137-2475.
\end{abstract}

The paper focuses on the category of verb and more specifically on support verbs which form compound predicates with abstract nouns. We discuss various functions of verbs within the language system and the role of the support verb in compound predicative expressions. We observe numerous cases where different verbs may co-occur with one and the same abstract noun. The substitution of one support verb for another partially or totally changes the meaning of the whole compound predicative expression. Analysis of such cases may be useful for studying different meanings of nouns.

\section{INTRODUCTION}

La catégorie du verbe est probablement la plus examinée en linguistique. Le caractère détaillé de cette recherche ne devrait pas étonner, vu l'intérêt manifesté par les chercheurs du XX-ème siècle aux grammaires formelles et par conséquent à la représentation de la phrase (unité de base) dans laquelle le verbe faisait cas d'élément central. Les spécialistes mettaient l'accent sur les propriétés sémanticogrammaticales de cette catégorie, en soulignant son rôle dans la structure d'emploi (c'est-à-dire dans une phrase simple où le verbe était vu comme noyau dont dépendaient les mots cooccurrents). Suivant cette approche, encore aujourd'hui, on commence l'analyse logique de la phrase le plus souvent à partir du verbe, en faisant de cette catégorie l'élément le plus important. La situation est semblable dans les études lexicographiques: si l'on entreprend des recherches visant la description systématique d'une classe d'unités lexicales d'une langue donnée, le verbe est toujours analysé en premier lieu. Pour diverses langues, il existe de nombreux dictionnaires sémantiques et syntaxiques des verbes. 
Dans ce travail, conformément au sujet principal du colloque (El verbo y su entorno: grammática, léxico, texto), nous présentons une fois de plus une étude de cette catégorie en nous limitant aux emplois dans lesquels le verbe apparaît à côté d'un prédicat nominal. Dans de tels contextes, le verbe est un élément constitutif d'un tour analytique qui, au niveau de la phrase simple, fait fonction de syntagme prédicatif. Nous essaierons de démontrer que le verbe joue un rôle important dans la définition sémantique de ces syntagmes.

\section{LE VERBE ET SA DESCRIPTION}

Malgré un nombre important d'études consacrées au verbe, il s'avère encore que les dictionnaires et les manuels de grammaire existant ne présentent pas exhaustivement tous ses aspects. Seule la lecture attentive des travaux linguistiques (malheureusement pour la plupart dispersés) permet de se rendre compte de toutes les fonctions de cette catégorie et de comprendre ce qu'on peut encore y apporter en guise de description.

Dans le discours, le verbe apparaît avant tout en tant qu'élément autonome (quand il est employé conformément à son sens). On dit alors qu'il est employé au sens «plein» et qu'il décrit ce que le sujet fait ou comment le sujet est. Dans de tels emplois, le verbe joue au niveau de la phrase le rôle de prédicat, c'està-dire il rend compte de la propriété ou de l'état du sujet (p.ex. dans les constructions intransitives du type : L'ascenseur fonctionne, Le Soleil brille, etc.) ou encore il informe sur la relation entre le sujet et les compléments régis (dans les constructions transitives du type : Paul lit un livre, Marc donne un cadeau à Marie etc.). Les exemples mentionnés montrent que le prédicat et le verbe font un.

Dans certaines langues, à part ces emplois typiques, il y en a d'autres (réguliers aussi) où le verbe est employé en tant qu'élément grammatical dans une structure plus complexe. Sa nature est alors fonctionnelle. En français, c'est par exemple le cas de la prédication composée au passé ou au futur où le verbe auxiliaire a pour fonction d'actualiser le prédicat verbal (cf. il a $v u$, il vient de voir, il aura $v u$, etc.). D'autres emplois où le verbe apparaît sans autonomie et où il n'obéit pas aux règles générales de constitution de phrases, c'est, par exemple, le cas des expressions figées (cf. il a perdu la tête, casser sa pipe, etc.) ou, encore, celui des verbes auxiliaires modaux qui créent une prédication avec un infinitif (cf. La mine doit sauter à 10 heures, si rien n'intervient entre-temps (Dubois, 1994 : 305). En plus, on distingue des verbes causatifs (cf. en français : causer, mettre, provoquer, occasionner, etc. ou en polonais: wprawić kogoś $w$ oshupienie, nadać komuśs wyglqd, wzbudzić w kimś gniew, etc.) qui expriment "que le sujet fait en sorte que d'autres fassent l'action... " (ibidem: 79). A la fin, on ne peut plus passer sous silence le dernier type de verbe (celui qui n'est pas encore régulièrement pris en compte par les grammaires, et encore moins enseigné au niveau scolaire malgré une 
littérature déjà riche en ce domaine), à savoir le verbe support dont la fonction est d'actualiser dans le discours les prédicats nominaux, adjectivaux ou autres (cf. en fr. : être d'une grande bonté, faire du sport, être bon, en pol. : być cztowiekiem wielkiej dobroci, uprawiać sport, być dobrym, etc.). Ici, le verbe a aussi le caractère d'outil grammatical. En simplifiant, on peut dire qu'il «conjugue » le nom (pour le français, voir p.ex. les travaux de : M. Gross, 1981 ; G. Gross \& R. Vivès (dir.), 1986 ; Ibrahim (dir.), 1996 ; dans la littérature polonaise, ce verbe est connu sous le nom de czasownik synsematyczny, podporowy, leksykalno-positkowy, operatorowy, etc., voir : Bogusławski, 1978 ; Topolińska (dir.), 1984 ; Buttler, 1988 ; Jędrzejko, 1998 ; Vetulani, 2000).

Dans la suite de cet article, nous nous concentrons seulement sur le dernier type, le verbe support (plus loin : VSup), en basant nos remarques sur les résultats que nous avons obtenus pendant le recensement des prédicats nominaux du polonais. Le recensement s'est fait de telle manière qu'à côté de chaque prédicat nous avons présenté entre autres un verbe (parfois plus) qui lui est propre. Ainsi, nous sommes arrivée à dénombrer non seulement les formes prédicatives simples, mais aussi les prédicats supportés par des verbes ce qui a permis d'avoir tout un ensemble d'unités composées, un type de tours analytiques qui, au niveau de la phrase, fonctionnent comme syntagmes prédicatifs. Ce sont des constructions comme: popetnić blą, uprawiać sport, wpaść w szal, wymierzyć mandat, wybuchną́ śmiechem, przypuścić szturm, wydać komendę, objać komendę, etc., Vetulani, 2000). Nous voulons démontrer ici que le rôle du verbe n'est pas à négliger dans ces syntagmes.

\section{LE SYNTAGME VERBO-NOMINAL EN TANT QUE PRÉDICAT}

Nous voulons traiter le verbe et le nom abstrait ensemble, comme si ces deux éléments constituaient une expression prédicative toute faite, une sorte de 'verbe analytique'. Ceci nous paraît légitime bien que, dans les travaux consacrés aux noms abstraits de la langue française (décrits selon les principes méthodologiques de lexique-grammaire), on considère que c'est le nom qui est le vrai prédicat sémantique et on dit du verbe qu'il est prédicativement vide. Un tel point de vue peut être justifié si l'on prend en considération ce que nous savons du prédicat dans l'acception logique; notamment que le prédicat est une catégorie décrivant le sujet : ses propriétés et ses relations avec les compléments. Ceci dit, on pourrait croire que, pour comprendre le sens d'un énoncé (le sens profond), il suffirait de comprendre ce à quoi renvoie le prédicat (comprendre ce qui se passe), dans notre cas, le

'Le terme est traduit du polonais (cf. czasownik analityczny). Vu ce que nous avons dit dans l'Introduction, on ne peut pas s'en étonner. En recherchant un terme adéquat pour ces tours analytiques, on a pensé en premier lieu au verbe. Dans la terminologie polonaise, fonctionne encore le terme de orzeczenie peryfrastyczne qui donncrait en français : 'verbe périphrastique'. 
prédicat nominal et non pas le verbe qui l'accompagne. Au niveau de la structure profonde de la langue, le fait d'avoir un prédicat verbal, nominal, adjectival ou un autre n'a pas d'importance. Par exemple, voyage signifie qu'il s'agit de 'voyager' (le prédicat nominal, du point de vue sémantique, ne diffère donc pas de son correspondant verbal voyager), désir signifie 'désirer' (tout comme désirer et dési$r e u x)^{2}$. Ces prédicats sont seulement différemment actualisés dans le discours. C'est là qu'intervient le VSup. Puisque le prédicat nominal ne peut pas se «conjuguer » à la manière d'un verbe, i] lui faut un support grammatical, le VSup en l'occurrence, qui fournit les informations sur l'agent engagé dans le procès (le nombre, le genre), de même que sur le temps et sur l'aspect du procès-même. Dans la détermination sémantique du syntagme verbo-nominal, le recours au seul prédicat pourrait paraître d'autant plus suffisant que les prédicats nominaux de la langue française sont très souvent actualisés par les VSup neutres (des formes sémantiquement pauvres qui n'enrichissent donc pas beaucoup le sens du syntagme entier) comme: être en voyage, avoir le désir (d'autres formes qui «supportent » régulièrement les prédicats sont, par exemple : faire, donner, etc.) Les remarques que nous venons de faire pourraient suggérer qu'il est pratiquement inintéressant de s'occuper de l'autre constituant du syn̨tagme, à savoir du VSup.

\section{QUEL USAGE PEUT-ON FAIRE DE LA NOTION DE VSUP?}

L'examen des emplois des prédicats nominaux en polonais nous amène à l'analyse des VSup. Il s'avère que le choix du verbe accompagnant un nom est peu prévisible. Parfois, on peut observer une forme neutre (comme en français) du type : robić (en fr. : faire; cf. robić porzqdek, robić zakupy, robić komplikacje) ou mieć (en fr. : avoir ; cf. mieć profit, mieć pożytek, mieć mdłości), mais très souvent, on rencontre une forme spécifique, propre au prédicat en question. Considérons, par exemple, ces cas: gadać glupstwa, pleść androny, palnać kazanie, pójiśc z wizyta wymierzyć sprawiedliwość, strzelić byka, zdradzać chęć, promieniować wesolościa tracić prowincjonalizmem, etc. Il y en a beaucoup d'autres. En polonais, chaque forme verbale, dans le contexte d'un prédicat nominal, est apte à remplir la fonction de VSup. On pourrait croire que ce grand nombre de verbes en fonction de « support » empêche la classification des prédicats du polonais ${ }^{3}$. Au contraire, nous considérons qu'une classification est possible, mais selon d'autres critères qu'en français [nous avons développé cette question ailleurs; Vetulani, 2000, 2003, à paraître (2)].

${ }^{2}$ A côté des prédicats sémantiquement identiques (mais différents au point de vue grammatical, cf., en fr. : lire / lecture, voyager / voyage, etc. ; en pol. ufać / ufność / ufny, dobroć / dobry, etc.), il existe des prédicats autonornes n'ayant pas de correspondants dans d'autres catégories grammaticales, Cf., en pol., des noms prédicatifs autonomes : nadzieja, scysja, klęska, etc.

${ }^{3}$ En français, les prédicats sont classifiés de telle manière qu'ils se regroupent en grands ensembles autour de quelques VSup seulement. 
Dans la suite, nous voulons présenter trois applications possibles de la notion de VSup. La première consiste en ce que le VSup se laisse exploiter comme indice des sens différents d'une même forme nominale. La deuxième application a lieu au moment ou un VSup remplace un autre, mais le prédicat garde son sens de base : le VSup est alors un outil qui permet d'adapter l'expression dans laquelle il se trouve à la situation de communication ou au type de discours. Enfin, le VSup s'avère aussi un outil commode dans la présentation du réseau dérivationnel des prédicats nominaux.

\subsection{LE VSUP ET LA POLYSÉMIE DES PRÉDICATS NOMINAUX}

Une forme prédicative peut être actualisée par des VSup différents (si c'est le cas, elle est polyvalente). En général, un choix d'éléments (un VSup donné + le prédicat en question) correspond à un sens. Cela signifie que le VSup peut être utile dans la reconnaissance sémantique du prédicat. Nous parlons de la situation où le remplacement du VSup par un autre VSup entraine le changement de sens de base du prédicat (plus loin, dans le chapitre 2.2., nous allons parler des remplacements semblables, mais sans conséquences au niveau du sens de base) ${ }^{4}$. Puisque nous avons déjà soulevé les problèmes liés à la polysémie des prédicats nominaux ailleurs [Vetulani, 2000, 2003 et à paraître (1)], nous nous limitons ici à la présentation de quelques exemples dans lesquels on voit que le seul recours au VSup suffit pour déterminer sémantiquement le prédicat. Le nouveau verbe est un indicateur du nouveau sens du prédicat. Comparons :

$\begin{array}{ll}\text { komenda: } & \text { 1. wydać komende (donner l'ordre de...) } \\ & \text { 2. objąć komende (avoir le commendement de...) } \\ \text { akcent: } & \text { 1. ktaść akcent (mettre l'accent sur...) } \\ & \text { 2. miéc akcent (avoir un accent...) } \\ \text { areszt: } & \text { 1. natożyć areszt (donner l'ordre d'arrestation) } \\ & \text { 2. odbywać areszt (être en état d'arrestation) } \\ \text { chód: } & \text { 1. mieć chody (être pistonné) } \\ & \text { 2. być na chodzie (fonctionner) } \\ \text { proces: } & \text { 1. wytoczyć proces (entreprendre un procès) } \\ & \text { 2. ulec procesowi (subir le processus de...) } \\ \text { mandat: } & \text { 1. wymierzyć mandat (donner une amende à qq.) } \\ & \text { 2. udzielić mandatu (donner un certain mandat à qq.) }\end{array}$

${ }^{4}$ Même s'il arrive encore que le groupe verbe + nom rcste polysémique, il cst facile de lcver l'ambiguîté grâce à l'analyse de différentes structures d'emploi de ce groupe. Il suffit d'observer alors d'autres éléments constitutifs dans ces structures, notamment la détermination du prédicat, le nombre d'arguments sélectionnés ou la manière dont les argurnents sont introduits : avec ou sans prépositions. 
dowcip: 1. zrobić dowcip (faire une plaisanterie)

2. opowiadać dowcipy (raconter des blagues)

3. mieć (jakiś) dowcip (être spirituel)

wiara: 1. mieć wiare $w$ (avoir confiance en...)

2. dawać wiare (croire)

3. przyjac wiare (faire une confession de foi)

wina: $\quad$ 1. ponosić wine (être coupable)

2. obarczyć winq (accuser)

\subsection{LE VSUP EN TANT QU'ÉLÉMENT ENRICHISSANT LE SENS DE BASE}

Dans toutes les langues, on observe des façons de parler variées selon l'interlocuteur, selon la situation ou selon le style employé. Bref, il y a une variation au niveau du système linguistique, il y a aussi une variation au niveau des syntagmes verbo-nominaux. Ceci est le résultat des besoins du locuteur qui, voulant adapter son discours à la situation de communication, peut employer différents VSup avec un prédicat donné, sans changer le sens de base du prédicat. Ainsi, autour d'une même forme, peuvent se regrouper en une famille des expressions partageant un élément sémantique (celui qui est fondamental, résidant dans le prédicat) et différant en sens additionnels apportés par les VSup. On appelle les verbes qui interviennent des variantes stylistiques. Le terme paraît naturel, vu le fait qu'il y a des usages neutres, standards, courants, recherchés, littéraires, familiers, populaires, relâchés, vulgaires, inhabituels, plaisants, affectifs, etc. En résumant, on pourrait dire que dans le discours, et par conséquent, dans les syntagmes verbo-nominaux, se reflète la société qui emploie la langue conformément à la situation. C'est pourquoi, les verbes apparaissant avec les prédicats sont très nombreux. Mais il ne faut pas envisager les prédicats dans leur ensemble pour voir les possibilités qui existent dans l'acceptation des formes verbales, de même que les possibilités qui existent dans la la création des expressions en général. Souvent, une forme suffit pour qu'on puisse se rendre compte de la productivité dans ce domaine. Les expressions créées autour d'un prédicat peuvent différer entre elles en degré de lexicalisation ou de stabilisation au niveau de la langue. A cause des changements constants dans le lexique, certaines expressions disparaissent, d'autres se maintiennent, les unes sont acceptées dans toutes les conditions, d'autres sont employées seulement par certains groupes sociaux ou dans certains types de discours et ainsi de suite.

Comparons quelques exemples pris du dictionnaire des noms prédicatifs du polonais (Vetulani, 2000), sans analyser à fond les différences sémantiques entre les syntagmes particuliers ayant pour base le même prédicat :

atak: przypuścić atak, ruszyć do ataku, przystqpić do ataku, przejść do ataku, dokonać ataku,

awantura: robic awantury, mieć awantury $z \ldots$, urzqdzać awantury, wplqtać się w awantury, 
choroba: wpaść $w$ chorobę, wpędzić się w chorobę, popaść w chorobę, zapaść na chorobe....,

ciagoty: mieć ciagoty do, zdradzać ciagoty, przejawiać ciagoty, czuć ciagoty,

cwat: puścić się w cwat, przejść w cwat, biec cwalem, gnać cwatem,

świczenie: zrobić ćwiczenie, wykonać ćwiczenie,

dezyderat: zglosić dezyderat, przedstawić swoje dezyderaty, wyrazić dezyderaty,

fanaberia: stroić fanaberie, wyprawiać fanaberie, mieć swoje fanaberie,

filipika: skierować filipike do..., wyglosić filipikę przeciwko..., wystapić z filipika

figiel: zrobić figla, sptatać figla

glód: zaznać glodu, cierpieć głód, odczuwać głód,

2watt: popetnić gwatt, dokonać gwattu, zadać gwatt,

kleska: ponieść klęske, doznać klęski, doświadczyć klęski, odczuwać klęskę.

koniec: dobiegać końca, mieć swój koniec, mieć się ku końcowi, chylić się ku końcowi

pomiar: dokonywać pomiaru, przeprowadzać pomiary, robić pomiary.

etc.

\subsection{LE RÉSEAU DÉRIVATIONNEL. DES NOMS PRÉDICATIFS}

Comme nous venons de voir, une expression prédicative donnée, ayant un sens de base, peut subir des transformations. Ces transformations s'effectuent à la position du VSup où les verbes s'échangeant provoquent soit le changement total du sens de l'expression, soit seulement une modification du sens de base. Mais pour avoir une image complète des échanges possibles, il faut encore prendre en considération la catégorie de l'aspect. En polonais, les verbes admettent des préfixes et suffixes (parfois, ce sont des formes supplétives) pour exprimer l'opposition entre l'aspect grammatical perfectif / imperfectif. Ainsi, un prédicat, s'il accepte un VSup, il accepte pour la plupart des cas les deux formes aspectuelles de ce verbe (bien que ce phénomène ne soit pas systématique car il se peut que le prédicat n'accepte qu'une des formes possibles) ${ }^{5}$.

Tout compte fait, nous pouvons envisager une présentation de l'organisation lexicale dans les syntagmes verbo-nominaux. Il semble que la meilleure méthode (vu sa précision et sa clarté) serait de recourir à une sorte de réseau dérivationnel proposé par Lewicki $(1996: 13)^{6}$ qui pourrait rendre compte de tous les phénomènes décrits.

Prenons en guise d'illustration le prédicat atak (attaque au sens de 'action d'attaquer, de commencer le combat') de la liste d'en haut (chap. 2.2.) et rajoutons

${ }^{5}$ Nous renonçons dans cet article à la discussion concernant l'expression de l'aspect en français et en polonais (nous avons soulevé ces problèmes dans Vetulani, 1994, 2000, 2003). Rappelons seulement que, dans le contexte des prédicats nominaux, on parle en français des variantes aspectuelles.

${ }^{6}$ Lewicki emploic le terme de siatka derywacyjna que nous avons ailleurs traduit en grille dérivationnelle (Vctulani, 2003 : 166), mais le terme de réseau dérivationnel nous paraî́ plus adéqual. 
aux VSup présentés, tous perfectifs, leurs correspondants imperfectifs. Il est facile d'y voir une régularité, cf. : przypuścić / przypuszczać atak, przystapić / przystępować do ataku, ruszyć / ruszać do ataku et ainsi de suite. En dehors des exemples analysés, le prédicat atak accepte encore le VSup być $+w$ (être + prép), cf. : być $w$ $a t a k u$. Ce verbe étant imperfectif n'a pas pourtant de correspondant perfectif. On pourrait y ajouter encore bywać (forme créee par le suffixe $(-y w-)$ de $b y c ́$ ), cf. : bywa $\dot{c} w$ ataku qui est aussi imperfective. Les expressions créées ainsi forment une famille dans laquelle chaque tour a le même sens de base : 'attaquer'. Pour ce sens-là, le prédicat $a t a k$ reçoit un réseau dérivationnel suivant :

atak: przypuscić atak/przypuszczać atak, ruszyć do ataku / ruszać do ataku przystapić do ataku / przystepować do ataku przejść do ataku / przechodzić do ataku dokonać ataku / dokonywać ataku być w ataku bywać w ataku

D'autres formations sont encore possibles (p.ex. iść do ataku), mais nous nous limitons ici aux emplois attestés dans le dictionnaire qui a servi de base pour le recensement. En tout cas, une telle présentation permet de rendre compte en même temps des variantes stylistiques et aspectuelles d'un prédicat (de toutes les formes qui «conjuguent» le prédicat). Précisons seulement que l'aspect grammatical perfectif / imperfectif se laisse observer dans la même ligne, tandis que l'aspect du point de vue général, exprimé à travers les variantes inchoatives, duratives, terminatives, ponctuelles, etc. est dispersé dans les lignes consécutives.

Nous savons aussi qu'une forme prédicative peut avoir des sens totalement différents. C'est aussi le cas du prédicat atak qui peut recevoir en plus le sens de 'maladie soudaine'. Pour ce deuxième sens, il faut prévoir un autre réseau, c.-à-d. former une autre famille d'expressions. Comparons :

atak: mieć atak (nerwowy, serca...)

miewać ataki (nerwowe, histerii..)

dostać / dostawać ataku (nerwowego, serca...)

En résumant : il y a autant de sens de base que de familles différentes.

\section{CONCLUSION}

La description sémantique approfondie des prédicats nominaux n'est pas possible sans l'analyse des verbes qui les accompagnent dans le discours. 


\section{BIBLIOGRAPHIE}

Bogusławski, A. (1978), Jednostki jezzyka a produkty jezykowe. Problem tzw. orzeczeń peryfrastycznych, Z zagadnień współczesnego języka polskiego, Prace Językoznawcze, 91, PAN, Ossolineum, 17-30.

Buttler, D. (1988), Leksykalne czasowniki posilkowe w konstrukcjach peryfrastycznych typu „wywrzeć wpływ" na przetomie XIX $i X X$ wieku, $Z$ problemów frazeologii polskiej i slowiańskiej, IV, 71-82.

Dubois, J. (dir.) (1994), Dictionnaire de linguistique et des sciences du langage. Paris: Larousse.

Gross, G. / Vivès, R. (dir.) (1986), Syntaxe des noms. Langue Française, 69.

Gross, M. (1981), Les bases empiriques de la notion de prédicat sémantique, Langages, 63, 7-52.

Ję drzejko, E. (1998), Slownik polskich zwrotów werbo-nominalnych. Zeszyt próbny. Warszawa.

Lewicki, A.M. (1976), Wprowadzenie do frazeologii syntaktycznej. Teoria zwrotu frazeologicznego. Katowice.

Lewicki, A.M. (1977), Zwroty frazeologiczne, czyli predykaty w formie skladników nieciaghych, Studja Gramatyczne, I, 135-143.

Lewicki, A.M. (1982), Stalość i zmienność zwiqzków frazeologicznych. Lublin.

Lewicki, A.M. (1996), Relacyjna siatka derywacyjna jako czynnik onomazjologicznego opisu frazeslogicznego, Problemy frazeologii europejskiej, I. Warszawa, 9-14.

Topolińska, Z. (dir.) (1984), Gramatyka wspólczesnego jezyka polskiego. Składnia. Warszawa.

Vetulani, G. (1994), Constructions à VSup + NPréd et l'aspect. Étude confrontative: Français Polonais. Akten des 28. Linguistischen Kolloquiums, Graz 1993, Tübingen: Max Niemeyer Verlag.

Vetulani, G. (1995), Rzeczowniki predykatywne w jezyku polskim, Studia Romanica Posnanicnsia, $\mathrm{XX}, 177 \cdot 197$.

Vetulani, G. (2000), Rzeczowniki predykatywne jezyka polskiego. W kierunku syntaktycznego stownika rzeczowników predykatywnych. Poznań.

Vetulani, G. (2003), Les collocations verbo-nominales et la traduction, Studia Romanica Posnaniensia, XXX, 160-168.

Vetulani, G. (à paraître 1), Collocations verbo-nominales: structure d'une entrée de dictionnaire, Akten des 36. Linguistischen Kolloquiums in Ljubljana 2001, Peter Lang.

Vetulani, G. (à parâtre 2), Repercussions des activités humaines dans les collocations verbonominales. 